Centro de Investigación en Metabolismo, Ejercicio y Salud (CIEMETS), Departamento de Educación Física, Universidad de la Frontera, Región de la Araucanía, Chile. ${ }^{2}$ Centro de Salud Familiar Público de Los Lagos, Región de Los Ríos,

Chile

aProfesor de Educación Física, MSc(C). Actividad Física y Salud.

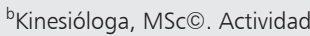
Física y Salud.

'Profesor de Educación Física, MSc. Entrenamiento Deportivo. ${ }^{\mathrm{d} N u t r i c i o n i s t a, ~ M S c}$.

${ }^{e}$ Nutricionista, MSc, PhD.

Financiamiento: Este estudio fue financiado con Proyecto DIUFRO DI12-007 de la Universidad de la Frontera. Los autores declaran que no existen conflictos de intereses en la fuente de financiamiento.

Recibido el 18 de junio de 2013 aceptado el 10 de enero de 2014.

Correspondencia a: Erik Díaz Bustos erick.diaz@ufrontera.c

\section{Ejercicio físico interválico de alta intensidad mejora el control glicémico y la capacidad aeróbica en pacientes con intolerancia a la glucosa}

\author{
RODRIGO MANCILLA ${ }^{1, \mathrm{a},}$, PAOLA TORRES ${ }^{1, \mathrm{~b}}$, CRISTIAN ÁLVAREZ $2, \mathrm{c}$, \\ INGRID SCHIFFERLI ${ }^{1, \mathrm{~d}}$, JORGE SAPUNAR ${ }^{1,}$, ERIK DÍAZ ${ }^{1, \mathrm{e}}$
}

Background: Proper exercise training modifies intra miocellular energy utilization, glucose transport and mitochondrial biogenesis. Aim: To determine the therapeutic effects of a high intensity intermittent training (HIIT) program on glucose homeostasis, physical fitness and body fat in glucose intolerant patients. Patients and Methods: Eighteen patients with overweight or obesity and glucose intolerance were invited to participate in an exercise program consisting in three sessions per week for 3 months. Ten participants aged $35 \pm 13$ years who attended $>26$ of the planned 36 sessions, were considered as adherent to exercise. The other eight participants aged $37 \pm 17$ years, who attended to a mean of 13 sessions, were considered as non-adherent. Both groups had similar body weight, body mass index, body fat, plasma glucose $2 \mathrm{~h}$ after an oral glucose load and maximal oxygen uptake. All these variables were measured at the end of exercise intervention. Each session consisted of 1 min exercise of cycling at maximal intensity until muscle fatigue followed by 2 min rest, repeated 10 times. Results: Among adherent participants, twelve weeks of HIIT improved significantly maximal oxygen uptake $(6.1+3.6 \mathrm{~mL} / \mathrm{kg} / \mathrm{min}$ or $24.6 \%)$, reduced $2 \mathrm{~h}$ post load blood glucose $(-33.7+47.9 \mathrm{mg} / \mathrm{dL}$ or $-12.5 \%)$ and body fat $(-4.3$ $+5.6 \mathrm{~kg})$. No significant changes were observed in the non-adherent group. Conclusions: HIIT exercise reduces blood glucose after an oral load in glucose intolerant patients.

(Rev Med Chile 2014; 142: 34-39)

Key words: Exercise; Glucose tolerance test; Obesity; Prediabetic state.

L a inactividad física es capaz de producir una reducción funcional de la masa muscular expresada en deterioro en la captación de la glucosa plasmática, de la oxidación de grasas y el aumento de grasa intramuscular, entre otros ${ }^{1}$. Para recuperar la funcionalidad, se requiere del efecto terapéutico del ejercicio sobre la masa muscular removiendo el efecto de diglicéridos y ceramidas que comprometen la cascada insulínica ${ }^{2}$, restituyendo la lipólisis ${ }^{3}$ y el metabolismo aeróbico de los sustratos ${ }^{4}$.

Como alternativa a las prescripciones tradicionales de ejercicio ${ }^{5,6}$, se han planteado los ejercicios de alta intensidad, intermitente y corta duración (HIIT), con reducción aguda en la curva de glicemia (AUC) continua de $24 \mathrm{~h}$, incrementando en 
$369 \%$ en el contenido de GLUT4 muscular ${ }^{7}$. El ejercicio HIIT es tiempo-eficiente y posee efectos intracelulares que remodelan metabólicamente el miocito ${ }^{8}$, incrementan la función endotelial ${ }^{9}$ y mejoran la capacidad cardio-respiratoria ${ }^{10}$, reduciendo la grasa corporal ${ }^{11,12}$.

Las bases moleculares del ejercicio HIIT se sustentan en el estrés energético de la célula y la disminución del glicógeno muscular. Dicho estímulo es sensado por la proteína kinasa $\mathrm{AMPK}^{13}$ lo que desencadena la activación de GLUT4, el aumento en la expresión génica de este transportador mejorando por ende la captación de glucosa, el incremento en la síntesis de proteínas del metabolismo lipídico y de biogénesis mitocondrial ${ }^{14,15}$.

Siendo la grasa corporal uno de los componentes principales en el compromiso funcional tisular, es de importancia mencionar que el ejercicio HIIT genera un estímulo adrenérgico ${ }^{16}$, que incrementa con cada repetición, estimulándose un efecto lipolítico de mayor potencia y duración ${ }^{4}$ Se liberan además IL-6 miocitaria y otros agentes lipolíticos, que incrementan este proceso en el tejido adiposo y muscular, entre otros ${ }^{17}$.

El propósito del estudio fue evaluar el efecto terapéutico de un programa de HIIT sobre la capacidad aeróbica, el control glicémico y la grasa corporal de pacientes intolerantes a la glucosa con sobrepeso u obesidad.

\section{Pacientes y Métodos}

Se invitó a la comunidad de la Universidad de la Frontera a participar de un programa de intervención de ejercicio físico y consejería nutricional.

Los criterios de inclusión fueron: a) IMC $\geq 25$ $\mathrm{y} \leq 43 \mathrm{~kg} / \mathrm{m}^{2}$; b) sedentarios; c) glicemia a los 120 min post carga oral de $75 \mathrm{~g}$ de glucosa $\geq 140 \mathrm{mg} / \mathrm{dl}$ (PTGO). Los pacientes tratados con metformina, suspendieron la medicación previo consentimiento del médico tratante. Los criterios de exclusión fueron, a) limitaciones físicas para realización de ejercicios y b) presencia de patologías respiratorias crónicas.

La muestra fueron 18 sujetos (12 mujeres y 6 hombres) con glicemia $\geq 140 \mathrm{mg} / \mathrm{dl} 120 \mathrm{~min}$ postPTGO y sobrepeso u obesidad. El tamaño de muestra calculado fueron 8 sujetos, empleando como variable dependiente el cambio en glicemia $^{18}$, $\alpha=0,05$ y poder $80 \%$. Considerando estudios previos $^{19}$, se aumentó la muestra considerando una adherencia de $50 \%$ en 3 meses. Cada sujeto actuó como control de sí mismo en la comparación pre-post intervención.

Los participantes se dividieron en dos grupos según asistencia a las sesiones de ejercicio; grupo adherente (GA, $\mathrm{n}=10$, edad $=35,4 \pm 13$ años) integrado por los pacientes que asistieron $>70 \%$ del total de 36 sesiones planificadas ( $\geq 26$ sesiones). La intervención de ejercicio se llevó a cabo en el CIEMETS, con frecuencia de 3 sesiones/semana (Lu/Miér/Vier) durante 3 meses. El grupo Noadherente (GNA) $(\mathrm{n}=8$, edad $=37,4 \pm 17$ años $)$ se conformó con los pacientes que abandonaron el estudio por razones laborales, estudio u otras personales, completando en promedio $13 \pm 4,6$ sesiones de ejercicio. Todos los pacientes expresaron buena tolerancia al ejercicio, sensación de fatiga muscular pero sin efectos adversos residuales. El estudio contó con la aprobación del Comité de Ética de la Facultad de Medicina de la Universidad de la Frontera, todo paciente firmó un consentimiento informado antes del estudio.

\section{Procedimientos}

Las mediciones de peso y $\%$ de grasa corporal ${ }^{20}$ se realizaron en un bioimpedanciómetro (Tani$\mathrm{ta}^{\circledR}$, China) condición descalza y ropa ligera. El sobrepeso y obesidad se clasificaron empleando el IMC $\left(\mathrm{kg} / \mathrm{m}^{2}\right)$.

La capacidad aeróbica se evaluó en cicloergómetro (Lode Corival Groeningen ${ }^{\circledR}$ ) y un analizador de gases (Ultima CPX Medgraphics, Minessota ${ }^{\circledR}$ ) previamente calibrado para volumen y gases de referencia. La capacidad aeróbica máxima $\left(\mathrm{VO}_{2 \mathrm{MAX}}\right)$ con monitoreo continuo de la frecuencia cardiaca (Polar, Finlandia) se efectuó mediante el test de Astrand modificado ${ }^{21}$.

Los pacientes fueron sometidos a una prueba de tolerancia a la glucosa oral (PTGO, ingesta de 75 g glucosa anhidra en $200 \mathrm{ml}$ de agua) luego de $\geq 12 \mathrm{~h}$ de ayuno. La glicemia se determinó mediante punción venosa a los 0, 30, 60, 90 y $120 \mathrm{~min}$ post-carga, usando el método de glucosa oxidasa (kits GOD-PAP de Diasys ${ }^{\circledR}$ ) en un autoanalizador Hitachi ${ }^{\circledR}$ modelo 911 (Boehringer Manheim, Alemania). En pacientes usando metformina, la PTGO se hizo una semana después de haberla suspendido. Los pacientes diagnosticados como diabéticos no tenían diagnóstico previo ni tratamiento. Todos los pacientes recibieron consejería nutricional individual por nutricionista, una vez 
Tabla 1. Características de los pacientes en estudio

\begin{tabular}{|c|c|c|c|c|}
\hline Variables & Test & Grupo adherente ${ }^{a}$ & Grupo no adherente ${ }^{b}$ & P interacción \\
\hline Pacientes & & $n=10$ & $\mathrm{n}=8$ & \\
\hline Edad (años) & Pre $_{0}$ & $35 \pm 14$ & $37 \pm 17$ & 0,787 \\
\hline Peso corporal (kg) & $\begin{array}{l}\text { Pre }_{0} \\
\text { Post }_{12} \\
\Delta \% \\
p=\end{array}$ & $\begin{array}{c}91,3 \pm 21 \\
90,2 \pm 20 \\
-1,1 \pm 2,6 \\
0,292\end{array}$ & $\begin{array}{c}79 \pm 9 \\
78,4 \pm 12 \\
-1,5 \pm 3,3 \\
0,191\end{array}$ & 0,180 \\
\hline IMC $\left(\mathrm{kg} / \mathrm{m}^{2}\right)$ & $\begin{array}{l}\text { Pre }_{0} \\
\text { Post }_{12} \\
\Delta \% \\
p=\end{array}$ & $\begin{array}{c}33,6 \pm 6 \\
32,8 \pm 6 \\
-0,8 \pm 1,8 \\
0,265\end{array}$ & $\begin{array}{c}29,2 \pm 4 \\
29,2 \pm 4 \\
0,03 \pm 2,0 \\
0,950\end{array}$ & 0,125 \\
\hline Género (F/M) & & $7 / 3$ & $5 / 3$ & \\
\hline Condición (NG/HG/DT2) & $\begin{array}{l}\text { Pre } \\
\text { Post }\end{array}$ & $\begin{array}{l}0 / 7 / 3 \\
6 / 4 / 0\end{array}$ & $\begin{array}{l}0 / 6 / 2 \\
2 / 4 / 2\end{array}$ & \\
\hline Metformina (con tto./ total) & & $3 / 10$ & $2 / 8$ & \\
\hline
\end{tabular}

por mes, consistente en recomendaciones de ingesta saludable, sin indicarse dieta hipocalórica.

Los pacientes se sometieron al ejercicio HIIT empleando una bicicleta estática (Oxford ${ }^{\circledR}$, BE2700) a una resistencia que indujera la fatiga muscular al cabo de 1 minuto de ejercicio y velocidad entre 30-40 km/h, empleando como referencia la frecuencia cardiaca máxima observada durante el test de $\mathrm{VO}_{2 \mathrm{MAX}}$, seguido de 2 min de descanso inactivo, repetido 10 veces. Los pacientes tuvieron supervisión personalizada y control de la frecuencia cardiaca al inicio y término de cada serie en cada sesión. Ambos grupos (GA y GNA) fueron evaluados al inicio y 3 meses post intervención.

\section{Análisis estadísticos}

Las diferencias pre-post intervención fueron analizadas mediante ANOVA con medidas repetidas y post-hoc de Bonferroni. La normalidad de las variables dependientes se evaluó mediante test de Shapiro-Wilks y Levene para la homogeneidad de las varianzas y homocedasticidad. Un margen de error alfa de $5 \%(\mathrm{p}<0,05)$ se usó en todas las comparaciones con el software (SPSS ${ }^{\circledR}$ versión 19.0).

\section{Resultados}

La Tabla 1 muestra las características al inicio y 3 meses post intervención para los 2 grupos de pacientes. No hubo diferencias significativas entre grupos por género, edad, condición clínica, uso de metformina, peso, IMC y masa grasa al inicio del estudio.

La Figura 1 muestra los cambios promedio en glicemia a los 120 min post PTGO y en la $\mathrm{VO}_{2 \mathrm{MAX}}$, también se pueden observar los cambios intrasujeto en el pre-post intervención del GA. Existieron diferencias estadísticamente significativas $(\mathrm{p}<0,05)$ sólo en el grupo GA, que determinó una disminución individual promedio de $34 \pm 48 \mathrm{mg} /$ $\mathrm{dL}$ en la glicemia post carga vs $6 \pm 32 \mathrm{mg} / \mathrm{dL}$ en el GNA. Las mismas comparaciones muestran que hubo un incremento de $24,6 \%$ en el $\mathrm{VO}_{2 \mathrm{MAX}}$, equivalentes a $6 \pm 3,6 \mathrm{~mL} / \mathrm{kg} / \mathrm{min}$ vs una disminución de $2 \pm 3 \mathrm{~mL} / \mathrm{kg} / \mathrm{min}$ en el grupo GNA.

La Figura 2 muestra las modificaciones en grasa corporal, observándose que solamente el grupo GA presentó reducciones significativas en este componente (GA: $-4,2 \pm 5,4 \mathrm{~kg}$ v/s GNA: $-1,2 \pm 3,6 \mathrm{~kg}$ ).

\section{Discusión}

Doce semanas de HIIT incrementaron la capacidad aeróbica, normalizaron en la mayoría de los pacientes la glicemia post carga y redujeron la masa grasa. La intervención permitió normalizar la glicemia en 6 de 10 pacientes del grupo GA, incluidos 2 de 3 pacientes diabéticos. Dos pacientes del GNA lograron también normalizar su glicemia. 
A

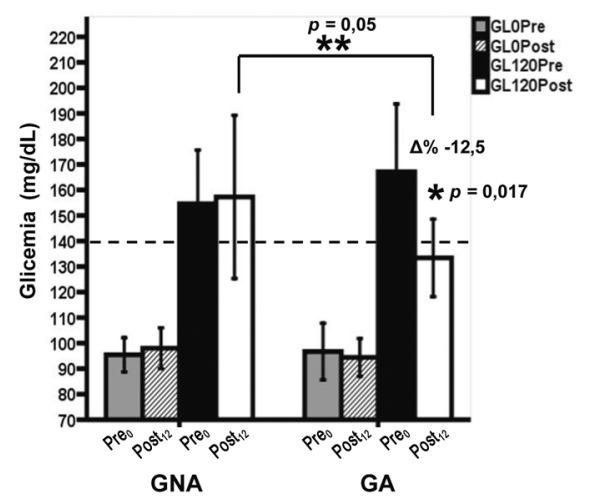

B

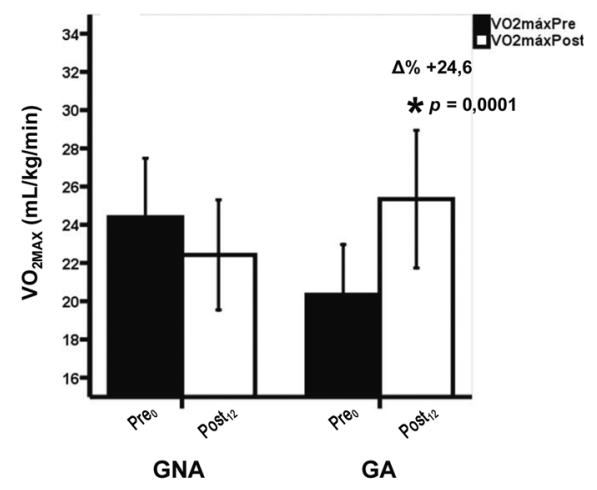

a

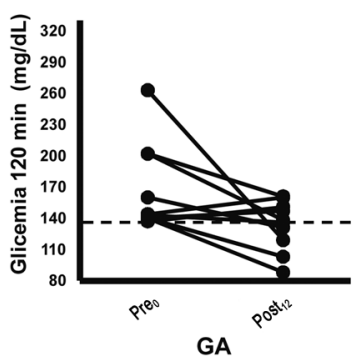

b

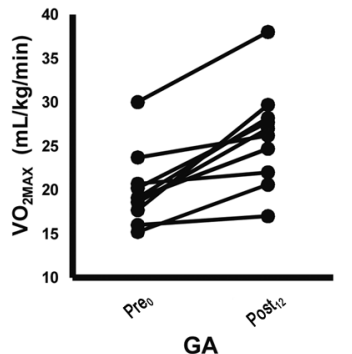

Figura 1. Cambios en la glicemia de ayuno, en la glicemia post carga de $75 \mathrm{~g}$ de glucosa y en el $\mathrm{VO}_{2 \mathrm{MAx}}$, en el grupo no adherente (GNA) y el grupo adherente (GA) sometido a 12 semanas de ejercicio físico tipo HIIT. A) indica glicemia basal (GLO) y a los 120 min post carga (GL120), valores se muestran en promedio \pm desviación estándar. a) indica datos individuales de glicemia a los 120 min post carga de $75 \mathrm{~g}$ de glucosa, en sujetos del grupo adherente. B) indica consumo máximo de oxígeno $\left(\mathrm{VO}_{2 \mathrm{MAX}}\right)$ durante un test incremental en bicicleta ergométrica en GNA y GA, valores se muestran en promedio \pm desviación estándar. b) indica datos individuales del $\mathrm{VO}_{2 \mathrm{MAX}}$ en el grupo adherente. Los cambios entre grupos fueron analizados mediante ANOVA de 1 vía, mientras que los cambios en el tiempo se analizaron con ANOVA de medidas repetidas. Pre $e_{0}$ indica antes de intervención, Post ${ }_{12}$ : indica después de 12 semanas de intervención. *indica diferencias significativas $(p<0,05)$ respecto a Pre 0 dentro de grupo. ${ }^{* *}$ indica diferencias significativas $(p<0,05)$ entre grupos.

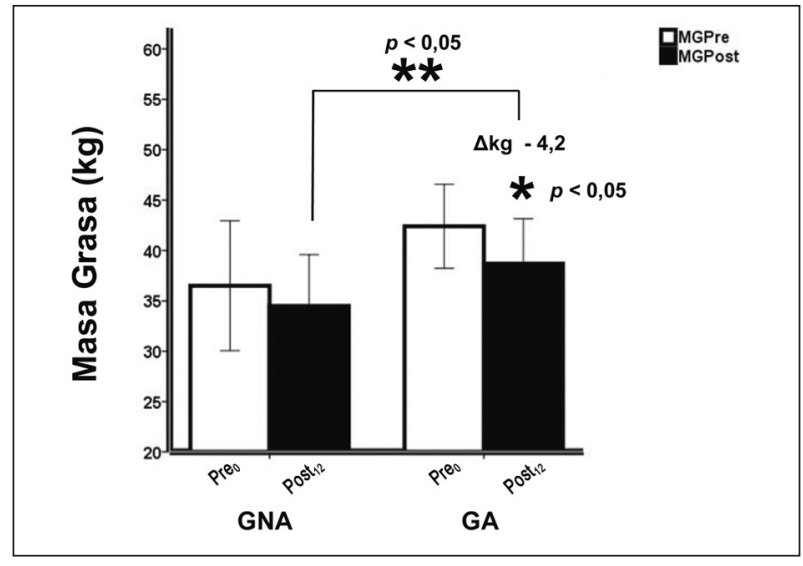

Figura 2. Modificación de la grasa corporal después de 12 semanas de intervención aplicando ejercicio físico tipo HIIT en dos grupos de pacientes según adherencia al programa de ejercicio. Grupos son descritos como GNA (Grupo No-adherente) y GA (Grupo Adherente). *indica diferencias significativas $(p<0,05)$ respecto a Pre $_{0}$ dentro de grupo. ${ }^{* *}$ indica diferencias significativas $(p<0,05)$ entre grupos en el mismo tiempo. 
La $\mathrm{VO}_{2 \mathrm{MAX}}$ mejoró $24,6 \%$ en el grupo GA, siendo mayores a la literatura; tanto con ejercicio de alta intensidad intermitente o continuo de intensidad moderada. Es el caso del estudio de Helgerud y cols ${ }^{22}$ que empleando cuatro modalidades de ejercicio, encontraron aumentos significativos (5,5\% y $7,2 \%)$ únicamente en los grupos que realizaron HIIT. En otro estudio, McPherson y $\mathrm{cols}^{23}$ muestra que el ejercicio HIIT produjo cambios de igual magnitud en el $\mathrm{VO}_{2 \mathrm{MAX}}$ en comparación con un ejercicio continuo (30-60 min) de intensidad moderada ( $11,5 \%$ y $12,5 \%$, respectivamente).

Respecto al empleo de ejercicio para mejorar las disglicemias ${ }^{24}$, se ha empleado una amplia gama de metodologías ${ }^{25,26}$, la mayoría sin lograr el mejoramiento de dicha condición. Tal es el caso de pacientes con diabetes mellitus tipo 2 (DM2) sometidos a ejercicio continuo de moderada intensidad durante $60 \mathrm{~min}$, que no lograron producir modificaciones en glicemia durante la prueba de PTGO $^{27}$.

En busca de un método más efectivo, los ejercicios HIIT se han convertido en una estrategia recurrente. Gillen y $\operatorname{cols}^{28}$, utilizando 1 minuto de ejercicio en cicloergómetro con 1 minuto de pausa, repetido 10 veces, encontró una mejoría significativa en el área bajo curva (AUC) de glicemia de $24 \mathrm{~h}$ en pacientes con DM2 con 1-6 sesiones de ejercicio ${ }^{7}$. Tales estudios con efectos agudos en las hiperglicemias diarias contrastan con el nuestro estudio donde la importancia clínica es haber logrado disminuir la hiperglicemia en 10/18 pacientes. Adicionalmente, 3 pacientes normalizaron la PTGO habiendo suspendido el uso de metformina cuyos efectos inhibidores de la función mitocondrial disminuyen los beneficios del ejercicio ${ }^{29,30}$. Al respecto, considerando que en Chile su administración está asegurada en todos los pacientes $^{31}$, debería garantizarse también el acceso a ejercicio terapéutico.

Otro resultado del presente estudio fue la reducción en la grasa corporal promedio $4,2+5,6 \mathrm{~kg}$ de grasa en el grupo GA (o su equivalente de $3,7 \pm$ 5,1 en porcentaje de grasa corporal). Tal variación supera los efectos en la homeostasis glicémica y grasa corporal encontrada en otros estudios que emplean HIIT en pacientes con sobrepeso, obesidad, síndrome metabólico o DM2 ${ }^{32}$, existiendo una amplia variedad en tiempo de ejercicio (desde 6 segundos a $4 \mathrm{~min}$ ) y pausa empleados, con intervenciones de 12-15 semanas de duración.
En conclusión, la modalidad de ejercicio HIIT del presente estudio recupera la glicemia y la capacidad de oxidación de grasa corporal en un lapso de 3 meses. Recomendamos su aplicación y modificar las estrategias de tratamiento.

Agradecimientos: A los profesores de Educación Física Álvaro Ponce y Verenna Dalmazzo y a la Nutricionista Ingrid Rivera por su generosa colaboración en el desarrollo del estudio.

\section{Referencias}

1. Thijssen D, Cable N, Green D. Impact of exercise training on arterial wall thickness in humans. Clin Sci 2012; 122: 311-22.

2. Coen P, Goodpaster B. Role of intramiocellular lipids in human health. Trends in endocrinology and metabolism 2012; 23 (8): 391-8.

3. Jocken J, Blaak E. Cathecolamine induced lipolysis in adipose tissue and skeletal muscle in obesity. Physiol Behav 2008; 94: 219-30.

4. Thompson D, Karpe F, Lafontan M, Frayn K. Physical activity and exercise in the regulation of human adipose tissue physiology. Physiol Rev 2012; 92: 157-91.

5. Garber C, Blissner B, Dechenes M, Franklin B, Lamonte $\mathrm{M}$, Lee I, et al. Quantity and quality of exercise for developing and maintaining cardiorespiratory musculoskeletal, and neuromotor fitness in apparently healthy adults: Guidance for prescribing exercise. Med Sci Sport Exerc 2011; 43 (7): 1334-59.

6. Colberg S, Sigal R, Fernhall B, Regensteiner J, Blissmer B, Rubin R, et al. Exercise and type 2 diabetes: The American College of Sport Medicine and the American Diabetes Asociation: joint position statement executive summary. Diabetes Care 2010; 33 (12): 2692-6.

7. Little, J, Gillen J, Percival M, Safdar A, Tarnopolsky M, Punthakee Z, et al. Low-volume high-intensity interval training reduces hyperglycemia and increases muscle mitochondrial capacity in patients with type 2 diabetes. Journal of Applied Physiology 2011; 111 (6): 1554-60.

8. Gibala M, Little J, MacDonald M, Hawley J. Physiological adaptations to low-volume high intensity interval training in health and disease. J Physiol 2012; 590 (Pt 5): 1077-84.

9. Ciolac E. High intensity interval training and hypertension: maximizing the benefits of exercise? Am J Cardiovasc Dis 2012; 2 (2): 102-10.

10. Hood M, Little J, Tarnopolsky M, Myslik F, Gibala M. Low-volume interval training improves muscle oxidative 
capacity in sedentary adults. Med Sci Sport Exerc 2011; 43 (10): 1849-56.

11. Álvarez C, Ramírez R, Flores M, Zúñiga C, Celis-Morales C. Efectos del ejercicio físico de alta intensidad y sobrecarga en parámetros de salud metabólica en mujeres sedentarias, pre-diabéticas con sobrepeso u obesidad. Rev Med Chile 2012; 140 (10): 1289-96.

12. Trapp E, Chrisholm D, Freund J, Boutcher S. The effects of high intensity intermittent exercise training on fat loss and fasting insulin levels of young women. Int J Obes 2008; 32 (4): 684-91.

13. Hardie DG. AMP-activated protein kinase: an energy sensor that regulates all aspects of cell function. Genes Dev 2011; 25 (18): 1895-908.

14. Little J, Safdar A, Wilkin G, Tarnopolsky M, Gibala M. A practical model of low-volume high-intensity interval training induces mitochondrial biogenesis in human skeletal muscle: potential mechanisms. J Physiol 2010; 588 (6): 1011-22.

15. Ritov V, Menshikova E, Azuma K, Wood R, Toledo F, Goodpaster B, et al. Deficiency of electron transport chain in human skeletal muscle mitochondria in type 2 diabetes mellitus and obesity. Am J Physiol Endocrinol Metab 2010; 298 (1): E49-58.

16. Bracken R, Linnane D, Brooks S. Plasma cathecolamines and nephrine response to brief intermittent maximal intensity exercise. Aminoacids 2009; 36: 209-17.

17. Pedersen B, Febbraio M. Muscle as an endocrine organ: focus on muscle derived interleukin-6. Physiol Rev 2008; 88: 1379-406.

18. Kasımay Ö, Ergen N, Bilsel S, Kaçar Ö, Deyneli O, Gogas $\mathrm{D}$, et al. Diet-supported aerobic exercise reduces blood endothelin-1 and nitric oxide levels in individuals with impaired glucose tolerance. J Clin Lipidol 2010; 4 (5): 427-34.

19. Jenkins N, Hagberg N. Aerobic training effects on glucose tolerance in prediabetic and normoglycemic humans. Med Sci Sports Exerc 2011; 43 (12): 2231-40.

20. Jebb S, Cole T, Doman D, Murgatroyd P, Prentice A. Evaluation of the novel Tanita body-fat analyser to measure body composition by comparison with a fourcompartment model. Br J Nutr 2000; 83: 115-22.

21. Astrand PO. Chapter 8, Physical performance. In: Astrand P.O, Rodahl K, Dahl H, Stromme S. (eds). Textbook of work physiology: Physiological basis of exercise ( $4^{\text {th }}$ ed). Champaign, IL, Human Kinetics 2003; p 237-72.
22. Helgerud J, Høydal K, Wang E, Karlsen T, Berg P, Bjerkaas $\mathrm{M}$, et al. Aerobic high-intensity intervals improve $\mathrm{VO}_{2 \max }$ more than moderate training. Med Sci Sports Exerc 2007; 39 (4): 665-71.

23. Macpherson R, Hazell T, Olver T, Paterson D, Lemon P. Run sprint interval training improves aerobic performance but not maximal cardiac output. Med Sci Sports Exerc 2011; 43 (1): 115-22.

24. Chen Z, Stephens T, Murthy S, Canny B, Hargreaves M, Witters L, et al. Effect of exercise intensity on skeletal muscle AMPK signaling in humans. Diabetes 2003; 52: 2205-12.

25. Umpierre D, Ribeiro P, Kramer C, Leitao C, Zucatti A, Azevedo M, et al. Physical activity advice only or structured exercise training and association with $\mathrm{HbA}_{1 \mathrm{c}}$ levels in type 2 diabetes. A systematic review and metaanalysis. JAMA 2011; 305 (17): 1790-99.

26. Snowling N, Hopkins W. Effect of different modes of exercise training on glucose control and risk factors for complications in type 2 diabetic patients. Diabetes Care 2006; 29: 2518-27.

27. Mikus C, Fairfax S, Libla J, Boyle LJ, Vianna L, Oberlin $\mathrm{D}$, et al. Seven days of aerobic exercise training improves conduit artery blood flow following glucose ingestion in patients with type 2 diabetes. J Appl Physiol 2011; 111 (3): 657-64.

28. Gillen J, Little J, Punthakee Z, Tarnopolsky M, Riddell M, Gibala M. Acute high-intensity interval exercise reduces the postprandial glucose response and prevalence of hyperglycaemia in patients with type 2 diabetes. Diabetes Obes Metab 2012; 14 (6): 575-7.

29. Viollet B, Guigas B, Sanz García N, Leclerc J, Foretz $\mathrm{M}$, Andreelli F. Cellular and molecular mechanisms of metformin: an overview. Clin Sci (Lond) 2012; 22 (6): 253-70.

30. Malin S, Nightingale J, Choi S, Chipkin S, Braun B. Metformin modifies the exercise training effects on risk factors for cardiovascular disease in impaired glucose tolerant adults. Obesity (Silver Spring). 2013; 21: 93-100.

31. Ministerio de Salud de Chile. Guías Clínicas de Diabetes Mellitus tipo 2, Santiago, MINSAL, 2010 (http://www. minsal.cl/portal/url/item/72213ed52c3e23d1e0400101 1f011398.pdf).

32. Boutcher S. High-intensity intermittent exercise and fat loss. J Obes. 2011; Article ID 868305 (doi: 10.1155/2011/868305. Epub 2010 Nov 24). 